\title{
Étude expérimentale du séchage à haute température d'une particule poreuse du bois
}

\author{
Souad Messai ${ }^{1, a}$, Didier Lecomte ${ }^{2}$, Jalila Sghaier ${ }^{3}$ et Ali Belghith ${ }^{3}$ \\ 1 Institut Supérieur des Sciences Appliquées et de Technologie de Gabès, Rue Omar Ibn El Khattab-6072, Gabès, Tunisie \\ 2 Centre RAPSODEE, UMR CNRS 2392, École des Mines d'Albi-Carmaux, Campus Jarlard, 81013 Albi CT Cedex 09, France \\ 3 Laboratoire d'Energétique et des Transferts Thermiques et Massiques, Faculté des Sciences de Tunis, Campus universitaire, \\ 1060 Tunis, Tunisie
}

Reçu le 18 juin 2012, accepté le 9 octobre 2012

Résumé - Une étude expérimentale du séchage convectif à haute température en vapeur d'eau surchauffée et du séchage à l'air d'une particule poreuse et sphérique du bois (hêtre) est présentée. Un dispositif de macro-TG, dans lequel une particule est suspendue et continuellement pesée au cours du séchage, est utilisé. En prenant deux valeurs de la vitesse du fluide séchant (vapeur d'eau surchauffée ou air), nous avons mesuré la teneur en eau et la température du produit au cours des deux procédés de séchage. Aussi, on a montré que le bois séché en vapeur d'eau surchauffée est nettement de qualité supérieure à celle obtenue à l'air. En effet, la particule du bois séchée en vapeur d'eau surchauffée garde sa couleur naturelle alors que celle séchée à l'air noircit. Le procédé de séchage en vapeur d'eau surchauffée répond alors au mieux à l'exigence la plus demandée par les industriels qui est qualité du produit. Parmi les applications possibles de séchage de ces particules est la fabrication du bois aggloméré.

Mots clés : Séchage / vapeur d'eau surchauffée / air / particule poreuse / haute température / qualité du produit

\begin{abstract}
Experimental study of wood porous particle drying at high temperature. The present work deals with an experimental study of convective high temperature superheated steam and hot air drying of wood porous spherical particle (beechwood). A "macro-TG" apparatus, where particle was suspended and continuously weighed during drying, was used. For two values of drying agent velocity (superheated steam or hot air), moisture content and product temperature were measured during the two drying process. Next, the product quality was demonstrated to be improved when superheated steam was the drying agent. Indeed, the wood particle dried with superheated steam keeps its natural color while that dried with hot air blackens. Then, superheated steam drying process provides the most demanding requirement of the industry which is product quality. Possible applications of this particles drying is the fabrication of agglomerated wood.
\end{abstract}

Key words: Drying / superheated steam / hot air / porous particle / high temperature / product quality

\section{Introduction}

Le séchage est une opération indispensable pour la mise en œuvre du bois. L'objectif du séchage de ce matériau à haute température est double : d'une part l'accélération des transferts, d'autre part les économies d'énergie. Il est couramment reconnu dans un procédé industriel que l'étape du séchage est la plus consommatrice d'énergie. Au cours de ces dernières années, malgré

\footnotetext{
a Auteur pour correspondance :

Souad.Messai@issatgb.rnu.tn
}

l'augmentation du prix de l'énergie, des contraintes plus strictes concernant la qualité, la pollution et la sécurité ont été imposées. Pour répondre à ces exigences et optimiser les dépenses liées à l'énergie, des actions de recherches doivent être menées afin de mieux gérer les procédés industriels. En effet, la plupart des procédés de séchage, basés sur la thermique, ont un faible rendement énergétique. Les procédés de séchage les plus courants par léchage à air chaud exigent théoriquement près de 700 kwh.m ${ }^{-3}$ d'eau évaporée; mais leur consommation réelle est 2 à 3 fois plus élevée. Pourtant, il est 
possible de réduire de manière significative les consommations énergétiques liées au séchage.

Quand le fluide séchant est la vapeur d'eau surchauffée (VES), au contact du produit à sécher, cette vapeur qui n'est pas saturée en eau se « désurchauffe » en cédant une partie de sa chaleur sensible, évaporant ainsi l'eau contenue dans le produit. Ce procédé ne convient pas aux produits sensibles à une très grande chaleur, mais se révèle avantageux pour ceux qui sont gorgés d'eau ou sensibles à l'oxydation. En effet, il est possible de récupérer l'énergie de la vapeur issue du produit sur le séchoir lui-même ou en dehors du séchoir. Ce procédé a en outre l'avantage de ne pas produire de gaz à effet de serre et son utilisation élimine les risques de feu et d'explosions.

$\mathrm{Au}$ cours du séchage à la VES, c'est la pression partielle de la vapeur d'eau du produit qui détermine les échanges de matière entre celui-ci et l'atmosphère qui l'entoure.

Lorsqu'il s'agit du séchage à l'air, la vitesse du séchage est liée à l'écart à l'équilibre dans des processus de diffusions externes ou internes du produit $[1,2]$.

Dans le cas du séchage à la VES, nous constatons un phénomène d'ébullition avec parfois une légère montée en pression à cause d'une résistance interne au flux de matière. Ce dernier est constitué de vapeur contenue dans le produit et évacuée vers une atmosphère vapeur entourant celui-ci. Il n'y a donc pas de résistance de diffusion à travers une couche limite entourant le produit, contrairement au séchage à l'air.

Le séchage en VES est une technologie intéressante qui fournit plusieurs avantages :

- Une économie d'énergie par rapport au séchage à l'air [3-5]. En effet, la recirculation de la VES permet d'évacuer uniquement la vapeur issue du séchage du produit.

- La récupération du solvant contenu dans le produit à sécher est simplifiée.

- La recompression mécanique de la vapeur dont le principe consiste à sécher par cascades successives à pression décroissante permet une récupération de l'énergie de la VES en sortie d'enceinte.

- L'élimination de l'air, et donc de l'oxygène, réduit aussi les risques d'incendie et d'explosion avec les produits combustibles à fort PCI (Pouvoir Calorifique Inférieur) tel que le charbon actif.

- La possibilité de récupérer le solvant réduit la pollution de l'environnement et les odeurs sont plus faciles à traiter.

- L'un des autres avantages, et qui est très exigé de nos jours suite à la concurrence, est la qualité de produit. Ce procédé du séchage garantit cette propriété. Nomura et Hyodo (1985) [6] observent, par exemple, une absence de durcissement de la surface du produit.

- Le contrôle du procédé est plus facile car les vitesses de séchage et l'humidité finale du produit sont facilement contrôlables en agissant sur la température de la vapeur.

Ce travail présente une étude expérimentale du séchage à haute température d'une particule poreuse sphérique du
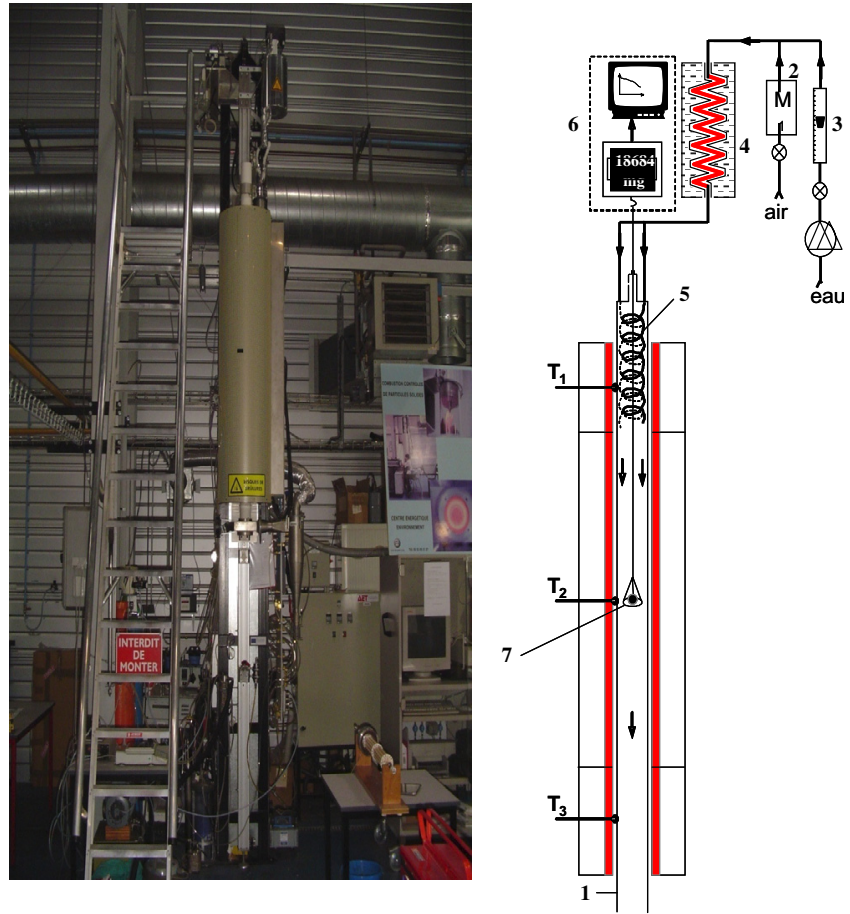

Fig. 1. Dispositif expérimental de la Macro-TG (centre RAPSODEE à Albi).

bois (hêtre) de diamètre $10 \mathrm{~mm}$. Dans le but de montrer que la qualité du produit séché à la VES est meilleure, nous avons utilisé deux fluides séchants : la VES et l'air.

\section{Dispositif expérimental}

Le principe général de la Macro-TG consiste à suspendre des particules dans un réacteur à pression atmosphérique et à une température contrôlée. La perte en masse est continuellement mesurée au cours du séchage. La figure 1 présente le dispositif expérimental de la MacroTG. Le réacteur est un tube de quartz de longueur $2 \mathrm{~m}$ (1), chauffée électriquement. L'atmosphère est générée par un flux d'air contrôlé par un débitmètre de masse (2) et un flux d'eau liquide contrôlé par un rotamètre (3) et dirigé vers le générateur de vapeur (4). La température de ce dernier est fixée à $200{ }^{\circ} \mathrm{C}$. Le mélange est alors conduit aux deux tubes spiraux (5) pour préchauffer le réacteur. Une balance (6), tenant le panier (7) qui contient les particules à sécher, est utilisée pour mesurer la perte en masse au cours du séchage.

Lorsque l'air sec est le fluide séchant, le générateur de vapeur n'est pas en service.

Pour des raisons pratiques du dispositif expérimental conçu pour la gazéfication du bois, on ne pouvait pas diminuer la température du séchage en dessous de $200^{\circ} \mathrm{C}$.

\section{Procédure expérimentale}

Les produits utilisés sont des particules sphériques et poreuses du bois (hêtre) de diamètre $10 \mathrm{~mm}$. Avant 


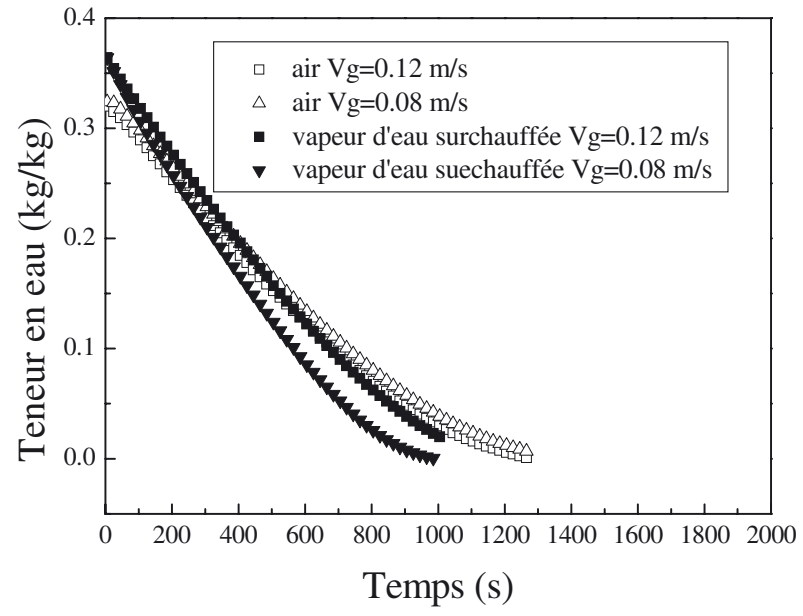

Fig. 2. Évolution de la teneur en eau d'une particule du bois au cours du séchage en vapeur d'eau surchauffée et à l'air.

d'effectuer les expériences, et pour assurer une saturation complète, ces particules sont immergées dans de l'eau distillée pendant quelques jours. La procédure expérimentale adoptée pour la mesure des cinétiques de séchage est la suivante : dans un premier temps, les conditions de séchage souhaitées, à savoir la température et la vitesse du fluide séchant, sont fixées. Une fois que les conditions expérimentales sont stabilisées, l'échantillon à sécher est placé dans le panier, relié à son tour à une balance, dans le but de mesurer la perte en masse au cours du séchage.

\section{Résultats expérimentaux}

La température du séchage étant maintenue à $200^{\circ} \mathrm{C}$, le panier dans lequel nous avons placé les particules du bois (nous avons placé plusieurs particules dans le panier afin de minimiser les erreurs expérimentales dans la mesure de la perte en masse) est accroché à une balance de façon à mesurer la perte en masse au cours du séchage. On fait varier la vitesse du fluide séchant $\left(V_{g}=0,08 \mathrm{~m} . \mathrm{s}^{-1}\right.$ et $V_{g}=0,12 \mathrm{~m} . \mathrm{s}^{-1}$ ) : la rapidité du séchage augmente quand la vitesse passe de 0,08 à $0,12 \mathrm{~m} \cdot \mathrm{s}^{-1}$ (Fig. 2). Ceci est essentiellement dû à une augmentation de la convection à la surface de la particule avec la vitesse du fluide séchant. Aussi, si on accélère les transferts, alors le temps du séchage est réduit. On note que la teneur en eau est définie comme étant le rapport de la masse d'eau sur la masse de la matière sèche contenue dans le produit. On remarque aussi que la cinétique de séchage à la VES est plus rapide qu'à l'air, pour les deux valeurs de la vitesse, ce qui implique qu'on est au-dessus de la température d'inversion. Cette température est définie comme celle pour laquelle le taux d'évaporation est le même pour des valeurs différentes de la vitesse du fluide séchant $[7,8]$.

Ces auteurs montrent que la variation de la température d'inversion, en fonction de la vitesse du fluide séchant, révèle l'existence d'un minimum lorsque cette température augmente. Donc cela confirme que pour des vitesses très faibles la température d'inversion est élevée,
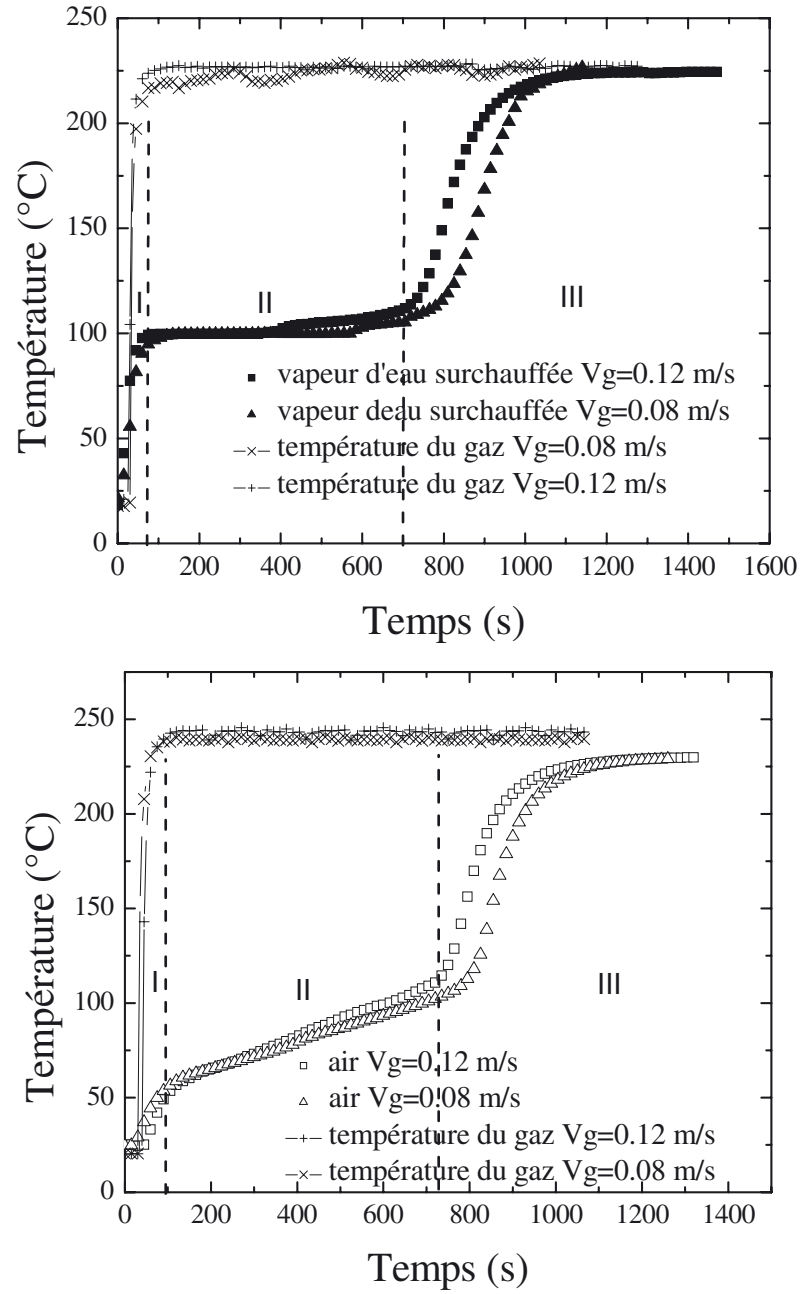

Fig. 3. Évolution de la température de la particule du bois et de la température du gaz au cours du séchage en vapeur d'eau surchauffée et à l'air.

ce qui explique la rapidité du séchage en VES par rapport au séchage à l'air dans ces conditions opératoires.

Les thermocouples utilisés sont de type K. Ils sont placés, l'un à l'intérieur des particules du bois pour mesurer la température du produit, et l'autre dans le tube de quartz pour mesurer la température du gaz (à l'intérieur du réacteur) au cours du séchage.

La figure 3 illustre les résultats obtenus lors du séchage d'une particule poreuse et sphérique du bois en VES et puis à l'air. On peut distinguer les trois phases classiques de séchage. Au cours de la phase II, le produit a une activité en eau à la surface qui est égale à 1. L'eau qui est évaporée à ce moment correspond à l'eau libre, c'est-à-dire non liée structurellement à l'intérieur du produit. Une migration de l'eau de l'intérieur du produit vers la périphérie remplace régulièrement cette eau évaporée, la vitesse de séchage est alors maximale et constante et dépend exclusivement des caractéristiques du fluide séchant.

L'énergie apportée par la vapeur sert uniquement à évaporer l'eau et non à chauffer le produit $[2,9]$. Ceci 

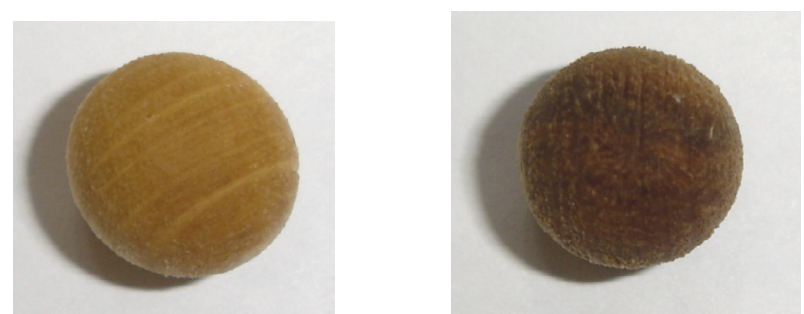

Fig. 4. Photos de particules du bois séchées en VES (à gauche) et à l'air (à droite).

n'est pas le cas pour le séchage à l'air. On remarque que la température du produit augmente.

Ceci montre l'importance des transferts pendant cette phase. En effet, dans le cas du séchage en VES, l'énergie apportée par ce procédé sert uniquement à évaporer l'eau contenue dans le produit (palier de $100{ }^{\circ} \mathrm{C}$ ). En revanche, dans le cas du séchage à l'air, cette énergie sert non seulement à l'évaporation de l'eau mais de plus à chauffer le produit. Nous constatons alors qu'une partie de cette énergie est utilisée à perte. À partir de cette observation, on peut déduire que le séchage en VES permet une économie appréciable d'énergie. On constate aussi que la température du gaz (dans le réacteur) dépasse la température du séchage dans le cas où le fluide séchant est l'air. Ceci peut être attribué à l'importance du transfert thermique par rayonnement dans le cas du séchage à l'air (milieu transparent) par rapport au séchage en VES (milieu semi-transparent).

La figure 4 illustre des photos des particules de bois après le séchage. On remarque que la particule du bois séchée à la VES garde sa couleur naturelle alors que celle séchée à l'air noircit et sa surface devient rugueuse. Il est évident que la qualité du produit séché en VES est meilleure que celle séché à l'air.

\section{Conclusion}

Une étude expérimentale du séchage en VES et à l'air a été mené. Un dispositif de «macro-TG » a été utilisé pour comparer les deux procédés de séchage. L'enjeu de ce dernier est d'obtenir du bois sec mais de qualité c'est-à-dire sécher vite mais en réduisant au maximum les défauts de séchage. Ceci n'est pas le cas pour le séchage à l'air à haute température. En effet, l'énergie fournie sert non seulement à évaporer l'eau du bois mais aussi à le réchauffer d'où le noircissement du bois séché à l'air. La qualité du bois séché en VES est nettement meilleure puisque la particule garde sa couleur naturelle après le séchage c'est donc une propriété esthétique garantie par ce procédé. De plus, le séchage du bois en vapeur d'eau surchauffée permet d'augmenter la durabilité naturelle du bois vis-à-vis des attaques de champignons et des insectes puisqu'il élimine des éléments à la base du développement de micro-organisme qui ont besoin d'un taux d'humidité minimal pour se développer. En effet, la teneur en eau d'équilibre (finale) obtenue pour le séchage en VES est inférieure à celle obtenue pour le séchage à l'air.

Les perspectives du séchage à haute température en VES s'annoncent parmi les procédés d'avenir à l'échelle industrielle.

\section{Références}

[1] M. Loncin, Génie Industriel Alimentaire, Aspects fondamentaux, Ed. Masson, Paris, 1976

[2] J.J. Bimbenet, Rapport sur le séchage des céréales, I.N.R.A., Montpellier, 1985

[3] P. Kumar, A.S. Mujumdar, Superheated steam drying: A bibliography, Drying Technology 8 (1990) 195-205

[4] C. Pronyk, S. Cenkowski, Superheated steam drying technology, Proceedings of ASAE Meeting Presentation, USA, 2003, paper number: RRV03-0014

[5] S. Devahastin, P. Suvarnakuta, S. Soponronnarit, A.S. Mujumdar, A comparative study of low-pressure superheated steam and vacuum drying of a heat-sensitive material, Drying Technology 22 (2004) 1845-1867

[6] T. Nomura, T. Hyodo, Behavior of inversion point temperature and new applications of superheated vapor drying, Proceedings of 4th International Drying Symposium, Washington, DC: Society of Chemical Engineers, 1985, pp. 804-809

[7] Z. Chen, W. Wu, P.K. Agarwal, Steam drying of coal. Part 1. Modeling the behavior of a single particle, Fuel 79 (2000) 961-973

[8] S. Messai, J. Sghaier, D. Lecomte, A. Belghith, Drying kinetics of porous spherical particle and the inversion temperature, Drying Technology-An Int. J. 26 (2008) $157-$ 167

[9] P. Perré, How to get a good material model for wood drying simulation? COST Action E15 Wood Drying Workshop, Edinburgh, Scotland, 1999, pp. 13-14 